\title{
Article \\ Existence Theory for Positive Iterative Solutions to a Type of Boundary Value Problem
}

Bo Sun

check for

updates

Citation: Sun, B. Existence Theory for Positive Iterative Solutions to a Type of Boundary Value Problem. Symmetry 2021, 13, 1585. https:// doi.org/10.3390/sym13091585

Academic Editor: Juan Luis García Guirao

Received: 27 July 2021

Accepted: 25 August 2021

Published: 28 August 2021

Publisher's Note: MDPI stays neutral with regard to jurisdictional claims in published maps and institutional affiliations.

Copyright: (C) 2021 by the author. Licensee MDPI, Basel, Switzerland. This article is an open access article distributed under the terms and conditions of the Creative Commons Attribution (CC BY) license (https:// creativecommons.org/licenses/by/ $4.0 /)$.
School of Statistics and Mathematics, Central University of Finance and Economics, Beijing 100081, China; sunbo@cufe.edu.cn

Abstract: We introduce some research results on a type of third-order boundary value problem for positive iterative solutions. The existence of solutions to these problems was proved using the monotone iterative technique. As an examination of the proposed method, an example to illustrate the effectiveness of our results was presented.

Keywords: successive iteration; positive solutions; third-order boundary value problem

\section{Introduction}

In the context of the intensification of research in various fields of applied mathematics and physics, it is necessary to discuss third-order differential equations in more detail. Third-order differential equation problems are fairly common; we often encounter them in the study of three-layer beams, the deflection of a curved beam having a constant or varying cross-section, gravity-driven flows, electromagnetic waves, and so on. For more on the applications of the third-order boundary value problems, see [1] and related topics.

We focus our attention on the third-order two-point boundary value problems. Some research has been undertaken into third-order two-point boundary value problems; see below for a summary.

The authors in [2] and the authors in [3] considered two different classes of third-order two-point boundary value problems respectively. They dealt with the problems in upper and lower solutions.

$\mathrm{Li}$ in [4] chose to discuss the existence of single and multiple positive solutions to a singular third-order two-point boundary value problem. Under certain assumptions, they have obtained some existence results by using Krasnoselskii's fixed point theorem.

Using the Leray-Schauder continuation principle, the authors obtained the existence of at least one positive solution for some third-order boundary value problems in [5].

The study focused on the upper and lower solution method because. The authors in [6] also thought that this method was interesting, so they developed it further. They discussed a class of third-order differential equations with mixed two-point boundary conditions. Some new existence results were obtained. Some applications were also presented.

Y. Sun, M. Zhao and S. Li were concerned with the existence of the monotone positive solution to a class of nonlinear third-order two-point boundary value problems in [7]. They gave us some suitable assumptions on $f$ and their tool was also a fixed point theorem. They obtained the existence of at least one positive solution to the problem they were concerned with.

In [8], the authors focused on a kind of third-order equation. They studied various twopoint boundary value problems for the equation they discussed. A variety of hypothetical conditions are taken into account. Then they obtained some rational results.

Again, we can study third-order boundary value problems in terms of giving the numerical solution. Ge and Zhang did this by using the barycentric rational interpolation collocation method in [9]. They focused on the matrix form of the problem they discussed. 
In the existing literature, most papers studied the existence of the solutions of various third-order boundary value problems by different types of techniques, for instance, see [10-16] and references therein. We focus our attention on computational methods. We undertook some preliminary work on this. For example, Ma, Du and Ge [17] and our papers $[18,19]$ considered some boundary value problems with the p-Laplace operator. It is worth mentioning that we discussed the Pseudo-Symmetric solutions which are based on symmetry solutions to a kind of boundary value problem in [18]. We chose the monotone iterative technique. Then we obtained some existence results for the problem we were concerned with.

From our point of view, on the one hand, it is worthwhile and interesting to discuss these kinds of problems with computational methods, especially when the nonlinear term is involved explicitly with the all-order derivatives. It is worth emphasizing that the problems with all-order derivatives not only bring a lot of difficulties in the discussion but also have strong practical application value. For example, in mechanics, the problems with all-order derivatives can describe more precisely the vibration of beams; see [1] and related topics.

On the other hand, the monotone iteration technique is an interesting and effective procedure for investigating the existence of solutions to nonlinear problems. For more information on the monotone iterative method, one may refer to $[10,20,21]$.

Motivated by the studies mentioned above, we will investigate the iteration and the existence of positive solutions to the following third-order two-point boundary value problem

$$
\begin{aligned}
& u^{\prime \prime \prime}(t)+q(t) f\left(t, u(t), u^{\prime}(t), u^{\prime \prime}(t)\right)=0, \quad 0<t<1, \\
& u(0)=u^{\prime}(1)=u^{\prime \prime}(0)=0,
\end{aligned}
$$

where $f(t, x, y, z) \in C\left([0,1] \times[0,+\infty) \times R^{2} \rightarrow[0,+\infty)\right), q(t)$ is a nonnegative continuous function defined on $(0,1), q(t) \not \equiv 0$ on any subinterval of $(0,1)$, and $\int_{0}^{1} q(t) d t<+\infty$. Our main tool is a monotone iterative technique improved from the classical monotone iterative technique of Amann [10].

We aim to obtain the existence of positive solutions for the problem we were concerned with. At the same time, we focus upon trying to construct some successive iterative schemes for approximating the solutions. We would like to emphasize that here we do not require the existence of lower and upper solutions in our discussion. Happily, the starting point of the successive iterative schemes is a known constant function or a simple quadratic function. Finally, we will give a numerical example to illustrate the applicability of our results. Then we will analyze the calculation model, give computation detail process and draw figures of the results. We remark that knowledge of how to find the solutions is probably most important from a numerical and application standpoint.

\section{Preliminaries}

Before reaching the main conclusions, we need to present some background information and preparation. Firstly, we present here some general definitions from cone theory.

Definition 1. Let E be a real Banach space. A nonempty closed set $P \subset E$ is said to be a cone provided that

(i) $a u+b v \in P$ for all $u, v \in P$ and all $a \geq 0, b \geq 0$, and

(ii) $u,-u \in P$ implies $u=0$.

Definition 2. The map $\alpha$ is said to be concave on $[0,1]$, if

$$
\alpha(t u+(1-t) v) \geq t \alpha(u)+(1-t) \alpha(v)
$$

for all $u, v \in[0,1]$ and $t \in[0,1]$. 
Secondly, let us do some preparatory work. Without loss of generality, the norm is defined as

$$
\|u\|:=\max \left\{\max _{0 \leq t \leq 1}|u(t)|, \max _{0 \leq t \leq 1}\left|u^{\prime}(t)\right|, \max _{0 \leq t \leq 1}\left|u^{\prime \prime}(t)\right|\right\} .
$$

in the Banach space $E=C^{2}[0,1]$. We introduce a symbol $E_{+}$and define the cone $P \subset E$ that

$$
\begin{gathered}
E_{+}=C_{+}^{2}[0,1]=\{u \in E \mid u(t) \geq 0, t \in[0,1]\} \\
P=\{u \in E \mid u(t) \geq 0, u \text { is concave and nondecreasing on }[0,1]\} .
\end{gathered}
$$

Lemma 1. Let $g \in L^{1}[0,1]$ then the boundary value problem

$$
\left\{\begin{array}{l}
u^{\prime \prime \prime}(t)+g(t)=0, \quad 0<t<1, \\
u(0)=u^{\prime}(1)=u^{\prime \prime}(0)=0 .
\end{array}\right.
$$

has a unique solution $u(t)=\int_{0}^{1} G(t, s) g(s) d s$, where

$$
G(t, s)=\left\{\begin{array}{lr}
t(1-s)-\frac{1}{2}(t-s)^{2}, & 0 \leq s \leq t \leq 1 \\
t(1-s), & 0 \leq t \leq s \leq 1
\end{array}\right.
$$

It is obvious that $0 \leq G(t, s) \leq G(1, s)=\frac{1}{2}\left(1-s^{2}\right)$.

Define an operator $T: P \rightarrow E$ by

$$
(T u)(t)=\int_{0}^{1} G(t, s) q(s) f\left(s, u(s), u^{\prime}(s), u^{\prime \prime}(s)\right) d s,
$$

According to the solving process of Lemma 1, if $u$ is a fixed point of $T$, then $T u=u$ means that $u=u(t)$ is a solution to boundary value problem (1). On the contrary, if $u=u(t)$ is a solution to the boundary value problem (1), we can get $T u=u$ which implies that $u$ is a fixed point of $T$. So the original problem of the solution is transformed into the fix point problem.

Lemma 2. $T: P \rightarrow P$ defined by (2) is completely continuous.

Proof. From the definition of $T$, we deduce that for each $u \in P$, there is $T u \in C^{2}[0,1]$ which satisfies (2). Because $(T u)^{\prime \prime}(t) \leq 0$ and $(T u)^{\prime}(t) \geq 0$, for $0 \leq t \leq 1$. Thus, $T u$ is concave and nondecreasing on $[0,1]$. Then, $(\mathrm{Tu})(t) \geq 0,0 \leq t \leq 1$. Hence, $T: P \rightarrow P$.

It is obvious that $T$ is continuous. Let $\Omega \subset P$ be an bounded set. It is easy to prove that $T \Omega$ is bounded and equi-continuous. The Arzela-Ascoli theorem guarantees that $T \Omega$ is relatively compact, which means $T$ is compact. Then, $T$ is completely continuous.

\section{The Existence and Iteration of Positive Solutions to (1)}

With the preparatory work in hand, let us obtain our main results step by step.

Theorem 1. Assume there exists a $>0$, such that the following $\left(H_{1}\right)-\left(H_{3}\right)$ hold, $\left(H_{1}\right): f\left(t, x_{1}, y_{1}, z_{1}\right) \leq f\left(t, x_{2}, y_{2}, z_{2}\right)$

for any $0 \leq t \leq 1,0 \leq x_{1} \leq x_{2} \leq a, 0 \leq y_{1} \leq y_{2} \leq a,-a \leq z_{2} \leq z_{1} \leq 0$;

$\left(H_{2}\right): \max _{0 \leq t \leq 1} f(t, a, a,-a) \leq \frac{a}{A}$, where $A=\int_{0}^{1} q(s) d s$

$\left(H_{3}\right): f(t, 0,0,0) \not \equiv 0$ for $0 \leq t \leq 1$.

then the boundary value problem (1) has at least one positive, nondecreasing and concave solution $w^{*}$ or $v^{*}$ with $\left\|w^{*}\right\| \leq a$ and $\left\|v^{*}\right\| \leq a$. There are some conclusions of $w^{*}$ and $v^{*}$ as follows

$$
\begin{aligned}
& w^{*}=\lim _{n \rightarrow \infty} w_{n}=\lim _{n \rightarrow \infty} T^{n} w_{0},\left(w^{*}\right)^{\prime}=\lim _{n \rightarrow \infty}\left(w_{n}\right)^{\prime}=\lim _{n \rightarrow \infty}\left(T^{n} w_{0}\right)^{\prime}, \\
& \left(w^{*}\right)^{\prime \prime}=\lim _{n \rightarrow \infty}\left(w_{n}\right)^{\prime \prime}=\lim _{n \rightarrow \infty}\left(T^{n} w_{0}\right)^{\prime \prime}, \text { where } w_{0}(t)=a t\left(1-\frac{t}{2}\right), \quad 0 \leq t \leq 1,
\end{aligned}
$$


and

$$
\begin{aligned}
& v^{*}=\lim _{n \rightarrow \infty} v_{n}=\lim _{n \rightarrow \infty} T^{n} v_{0},\left(v^{*}\right)^{\prime}=\lim _{n \rightarrow \infty}\left(v_{n}\right)^{\prime}=\lim _{n \rightarrow \infty}\left(T^{n} v_{0}\right)^{\prime}, \\
& \left(v^{*}\right)^{\prime \prime}=\lim _{n \rightarrow \infty}\left(v_{n}\right)^{\prime \prime}=\lim _{n \rightarrow \infty}\left(T^{n} v_{0}\right)^{\prime \prime}, \text { where } v_{0}(t)=0, \quad 0 \leq t \leq 1,
\end{aligned}
$$

where $(T u)(t)$ is defined by (3).

The iterative schemes in this theorem are $w_{0}(t)=$ at $\left(1-\frac{t}{2}\right), w_{n+1}=T w_{n}=T^{n} w_{0}$, $n=0,1,2 \ldots$ which starts off with a known simple quadratic function and $v_{0}(t)=0, v_{n+1}=$ $T v_{n}=T^{n} v_{0}, n=0,1,2 \ldots$ which starts off with the zero function.

Proof. We denote $\overline{P_{a}}=\{u \in P \mid\|u\| \leq a\}$. Firstly, we will prove that $T: \overline{P_{a}} \rightarrow \overline{P_{a}}$.

If $u \in \overline{P_{a}}$, by $\left(H_{1}\right)$ and $\left(H_{2}\right)$ we have

$0 \leq f\left(t, u(t), u^{\prime}(t), u^{\prime \prime}(t)\right) \leq f(t, a, a,-a) \leq \max _{0 \leq t \leq 1} f(t, a, a,-a) \leq \frac{a}{A}$, for $0 \leq t \leq 1$.

Since

$$
\begin{aligned}
& (T u)(1) \leq \frac{a}{A} \frac{1}{2} \int_{0}^{1} q(s) d s<a,(T u)^{\prime}(0) \leq \frac{a}{A} \int_{0}^{1} q(s) d s=a, \\
& -(T u)^{\prime \prime}(1) \leq \frac{a}{A} \int_{0}^{1} q(s) d s=a .
\end{aligned}
$$

Hence, we assert that $T: \overline{P_{a}} \rightarrow \overline{P_{a}}$.

By $\left(H_{1}\right)$ we have, for any $u_{i} \in P(i=1,2)$ with $u_{1} \leq u_{2}, u_{1}^{\prime} \leq u_{2}^{\prime}$ and $u_{1}^{\prime \prime} \geq u_{2}^{\prime \prime}$. Then by (2) and (3), we can easily get $T u_{1} \leq T u_{2},\left(T u_{1}\right)^{\prime} \leq\left(T u_{2}\right)^{\prime}$ and $\left(T u_{1}\right)^{\prime \prime} \geq\left(T u_{2}\right)^{\prime \prime}$.

Let $w_{0}(t)=a t\left(1-\frac{t}{2}\right), 0 \leq t \leq 1$, then $w_{0}(t) \in \overline{P_{a}}$. Let $w_{1}=T w_{0}$, then $w_{1} \in \overline{P_{a}}$. We denote $w_{n+1}=T w_{n}, n=0,1,2 \ldots$ Then we have $w_{n} \subseteq \overline{P_{a}}, n=1,2, \ldots$ Due to the complete continuity of $T,\left\{w_{n}\right\}_{n=1}^{\infty}$ is asserted a sequentially compact set.

Because

$$
\begin{aligned}
w_{1}(t) & =T w_{0}(t)=\int_{0}^{1} G(t, s) q(s) f\left(s, w_{0}(s), w_{0}^{\prime}(s), w_{0}^{\prime \prime}(s)\right) d s \leq a t\left(1-\frac{t}{2}\right)=w_{0}(t), 0 \leq t \leq 1, \\
w_{1}^{\prime}(t) & =\left(T w_{0}\right)^{\prime}(t) \\
& =\int_{0}^{t}(s-t) q(s) f\left(s, w_{0}(s), w_{0}^{\prime}(s), w_{0}^{\prime \prime}(s)\right) d s+\int_{0}^{1}(1-s) q(s) f\left(s, w_{0}(s), w_{0}^{\prime}(s), w_{0}^{\prime \prime}(s)\right) d s \\
& \leq a(1-t)=w_{0}^{\prime}(t), \quad 0 \leq t \leq 1,
\end{aligned}
$$

and

$$
w_{1}^{\prime \prime}(t)=\left(T w_{0}\right)^{\prime \prime}(t)=-\int_{0}^{t} q(s) f\left(s, w_{0}(s), w_{0}^{\prime}(s), w_{0}^{\prime \prime}(s)\right) d s \geq-a=w_{0}^{\prime \prime}(t), \quad 0 \leq t \leq 1 .
$$

We obtain that

$$
\begin{aligned}
& w_{2}(t)=T w_{1}(t) \leq T w_{0}(t)=w_{1}(t), \quad w_{2}^{\prime}(t)=\left(T w_{1}\right)^{\prime}(t) \leq\left(T w_{0}\right)^{\prime}(t)=w_{1}^{\prime}(t), \\
& w_{2}^{\prime \prime}(t)=\left(T w_{1}\right)^{\prime \prime}(t) \geq\left(T w_{0}\right)^{\prime \prime}(t)=w_{1}^{\prime \prime}(t), 0 \leq t \leq 1
\end{aligned}
$$

Compute continuously according to the iteration rule and by the induction, then

$$
w_{n+1} \leq w_{n}, \quad w_{n+1}^{\prime}(t) \leq w_{n}^{\prime}(t), \quad w_{n+1}^{\prime \prime}(t) \geq w_{n}^{\prime \prime}(t), \quad 0 \leq t \leq 1, \quad n=0,1,2 \ldots
$$

Then we can show there exists $w^{*} \in \overline{P_{a}}$ that satisfies $w_{n} \rightarrow w^{*}$. By applying the continuity of $T$ and the iteration of $w_{n+1}=T w_{n}$ in combination, we obtain $T w^{*}=w^{*}$.

The other iteration direction is start off with the zero function. We choose $v_{0}(t)=$ $0,0 \leq t \leq 1$, then $v_{0}(t) \in \overline{P_{a}}$. Let $v_{1}=T v_{0}$, then $v_{1} \in \overline{P_{a}}$. We denote $v_{n+1}=T v_{n}, n=$ $0,1,2 \ldots$ Then we have $v_{n} \subseteq \overline{P_{a}}, n=1,2, \ldots$ Since $T$ is completely continuous, we assert that $\left\{v_{n}\right\}_{n=1}^{\infty}$ is a sequentially compact set. 
Since $v_{1}=T v_{0} \in \overline{P_{a}}$, we have $v_{1}(t)=T v_{0}(t) \geq 0, v_{1}^{\prime}(t)=T v_{0}^{\prime}(t) \geq 0, v_{1}^{\prime \prime}(t)=$ $\left(T v_{0}\right)^{\prime \prime}(t) \leq 0$, for $0 \leq t \leq 1$. Then $v_{2}(t) \geq v_{1}(t), v_{2}^{\prime}(t) \geq v_{1}^{\prime}(t), v_{2}^{\prime \prime}(t) \leq v_{1}^{\prime \prime}(t)$, for $0 \leq$ $t \leq 1$. By an induction argument similar to the above we obtain

$$
v_{n+1} \geq v_{n}, \quad v_{n+1}^{\prime}(t) \geq v_{n}^{\prime}(t), \quad v_{n+1}^{\prime \prime}(t) \leq v_{n}^{\prime \prime}(t), \quad 0 \leq t \leq 1, \quad n=0,1,2 \ldots
$$

Hence there exists $v^{*} \in \overline{P_{a}}$ such that $v_{n} \rightarrow v^{*}$. Applying the continuity of $T$ and $v_{n+1}=T v_{n}$, we get $T v^{*}=v^{*}$.

If $f(t, 0,0,0) \not \equiv 0,0 \leq t \leq 1$, then the zero function is not the solution of (1). Thus $\max _{0 \leq t \leq 1}\left|v^{*}(t)\right|>0$, we have $v^{*}>0$, for $0<t<1$.

From the previous discussion, if we find the fixed point of $T$ in $P$, we get the solution to (1). Hence, we can conclude that that the boundary value problem (1) has at least one positive nondecreasing and concave solution $w^{*}$ or $v^{*}$.

Furthermore, if $\lim _{n \rightarrow \infty} w_{n} \neq \lim _{n \rightarrow \infty} v_{n}$, then $w^{*}$ and $v^{*}$ are different. We obtain problem (1) has two positive nondecreasing and concave solutions. The other case is $\lim _{n \rightarrow \infty} w_{n}=\lim _{n \rightarrow \infty} v_{n}$, then $w^{*}=v^{*}$ which means the two values coincide and problem (1) has a positive nondecreasing and concave solution. Anyway, the problem (1) has at least one positive nondecreasing and concave solution.

The proof is completed.

The following corollary follows easily.

Corollary 1. Assume that $\left(H_{1}\right)$ and $\left(H_{3}\right)$ hold, and there exists $a>0$, such that $\left(H_{4}\right): \underline{\lim }_{\ell \rightarrow+\infty} \max _{0 \leq t \leq 1} \frac{f(t, \ell, a,-a)}{\ell} \leq \frac{1}{A}$, (particularly, $\underline{\lim }_{\ell \rightarrow+\infty} \max _{0 \leq t \leq 1} \frac{f(t, \ell, a,-a)}{\ell}=0$ ). Then the boundary value problem (1) has at least one positive, nondecreasing and concave solution $w^{*}$ or $v^{*}$. Consequently, the relevant conclusions about $w^{*}$ and $v^{*}$ in Theorem 1 hold.

\section{Example}

Example 1. Let $q(t)=1$, we consider the following boundary value problem

$$
\begin{gathered}
u^{\prime \prime \prime}(t)+f\left(t, u(t), u^{\prime}(t), u^{\prime \prime}(t)\right)=0,0<t<1, \\
u(0)=u^{\prime}(1)=u^{\prime \prime}(0)=0,
\end{gathered}
$$

where $f(t, x, y, z)=\frac{1}{4} t^{2}+\frac{1}{4} x+\frac{1}{8} y^{2}-\frac{1}{4} z$. Choose $a=2$, and we have $A=1$.

So by Theorem 1, the boundary value problem (4) and (5) has at least one positive, nondecreasing and concave solution $w^{*}$ or $v^{*}$ with $\left\|w^{*}\right\| \leq 2$ and $\left\|v^{*}\right\| \leq 2$, such that

$$
\begin{aligned}
& w^{*}=\lim _{n \rightarrow \infty} w_{n}=\lim _{n \rightarrow \infty} T^{n} w_{0}, \quad\left(w^{*}\right)^{\prime}=\lim _{n \rightarrow \infty}\left(w_{n}\right)^{\prime}=\lim _{n \rightarrow \infty}\left(T^{n} w_{0}\right)^{\prime}, \\
& \left(w^{*}\right)^{\prime \prime}=\lim _{n \rightarrow \infty}\left(w_{n}\right)^{\prime \prime}=\lim _{n \rightarrow \infty}\left(T^{n} w_{0}\right)^{\prime \prime}, \text { where } w_{0}(t)=2 t-t^{2}, \quad 0 \leq t \leq 1,
\end{aligned}
$$

and

$$
\begin{aligned}
& v^{*}=\lim _{n \rightarrow \infty} v_{n}=\lim _{n \rightarrow \infty} T^{n} v_{0},\left(v^{*}\right)^{\prime}=\lim _{n \rightarrow \infty}\left(v_{n}\right)^{\prime}=\lim _{n \rightarrow \infty}\left(T^{n} v_{0}\right)^{\prime}, \\
& \left(v^{*}\right)^{\prime \prime}=\lim _{n \rightarrow \infty}\left(v_{n}\right)^{\prime \prime}=\lim _{n \rightarrow \infty}\left(T^{n} v_{0}\right)^{\prime \prime}, \text { where } v_{0}(t)=0, \quad 0 \leq t \leq 1,
\end{aligned}
$$

where $(T u)(t)$ is defined by (3).

For $n=0,1,2 \ldots$ The two iterative schemes are 


$$
\begin{aligned}
& w_{0}(t)=2 t-t^{2}, \quad 0 \leq t \leq 1, \\
& w_{1}(t)=-\frac{1}{120} t^{5}+\frac{1}{48} t^{4}-\frac{1}{6} t^{3}+\frac{11}{24} t, \quad 0 \leq t \leq 1, \\
& w_{2}(t)=-\frac{1}{4561920} t^{11}+\frac{1}{829440} t^{10}-\frac{1}{82944} t^{9}+\frac{1}{26880} t^{8}-\frac{73}{483840} t^{7}-\frac{11}{138240} t^{6} \\
& -\frac{5}{2304} t^{5}-\frac{35}{2304} t^{4}-\frac{121}{27648} t^{3}+\frac{24989}{290304} t, \quad 0 \leq t \leq 1 \\
& w_{3}(t)=-\frac{t^{23}}{14620753605427200}+\frac{t^{22}}{1271369878732800}-\frac{23 t^{21}}{2196002517811200}+\frac{271 t^{20}}{3660004196352000} \\
& -\frac{9649 t^{19}}{18666021401395200}+\frac{2201 t^{18}}{982422179020800}-\frac{823 t^{17}}{74912366592000}+\frac{5503 t^{16}}{299649466368000} \\
& -\ldots \ldots \ldots-\frac{807311 t^{6}}{11466178560}-\frac{632025743 t^{5}}{128421199872}-\frac{8153 t^{4}}{6967296}-\frac{624450121 t^{3}}{4045267795968} \\
& +\frac{5622836449329950699 t}{186846874227965952000}, \quad 0 \leq t \leq 1 \text {, } \\
& w_{4}(t)=-\frac{t^{47}}{314515486692275039735441994547200000}+\frac{t^{46}}{13383637731586171903635829555200000} \\
& -\frac{173 t^{45}}{118852522029411983100766008115200000}+\frac{571 t^{44}}{29581072149542538016190650908672000} \\
& 31616289735913447234529323165770588601 t^{3} \\
& \text { - } \cdots \cdots \cdots-\frac{31616289735913447234529323165770588601 t^{3}}{1675764211620543699419355421020782592000000} \\
& +\frac{160511358248153446347381073284028009201404114366497 t}{6780718732903555345790847898231140300789645312000000}, \quad 0 \leq t \leq 1, \\
& w_{n+1}(t)=\left(T w_{n}\right)(t)=\int_{0}^{1} G(t, s)\left(\frac{1}{4} s^{2}+\frac{1}{4} w_{n}(s)+\frac{1}{8} w_{n}^{\prime 2}(s)-\frac{1}{4} w_{n}^{\prime \prime}(s)\right) d s, \quad 0 \leq t \leq 1 . \\
& v_{0}(t)=0, \\
& v_{1}(t)=-\frac{1}{240} t^{5}+\frac{1}{48} t, \quad 0 \leq t \leq 1 \text {, } \\
& v_{2}(t)=-\frac{1}{18247680} t^{11}+\frac{1}{322560} t^{8}+\frac{1}{1935360} t^{7}-\frac{1}{5760} t^{6}-\frac{1}{240} t^{5}-\frac{1}{4068} t^{4} \\
& -\frac{1}{110592} t^{3}+\frac{33011}{1451520} t, \quad 0 \leq t \leq 1 \text {, } \\
& v_{3}(t)=-\frac{t^{23}}{233932057686835200}+\frac{t^{20}}{1830002098176000}+\frac{t^{19}}{10666297943654400}-\frac{t^{18}}{31188005683200} \\
& -\frac{251 t^{17}}{318377558016000}-\frac{41 t^{16}}{898948399104000}+\frac{55247 t^{15}}{23372658376704000}+\frac{3919 t^{14}}{59513713459200} \\
& -\cdots \cdots \cdots-\frac{132359 t^{4}}{557383680}-\frac{1089726121 t^{3}}{101131694899200}+\frac{251944745719415081 t}{10990992601645056000}, \quad 0 \leq t \leq 1,
\end{aligned}
$$




$$
\begin{aligned}
v_{4}(t)= & -\frac{t^{47}}{80515964593222410172273150604083200000}+\frac{t^{44}}{295810721495425380161906509086720000} \\
& +\frac{t^{43}}{16911557584331769512468804411484160000}-\frac{t^{42}}{4855457244999072498555499315200000} \\
& +\cdots \quad \ldots \quad \cdots-\frac{63476154895620723780956748770236561 t^{3}}{5798492081731985119098115643670528000000} \\
& +\frac{49776339527441954834684459870069552303067862246322909 t}{2169829994529137710653071327433964896252686499840000000}, \quad 0 \leq t \leq 1, \\
& \ldots \quad \cdots \quad \cdots \quad \cdots \\
v_{n+1}(t) & =\left(T v_{n}\right)(t)=\int_{0}^{1} G(t, s)\left(\frac{1}{4} s^{2}+\frac{1}{4} w_{n}(s)+\frac{1}{8} w_{n}^{\prime 2}(s)-\frac{1}{4} w_{n}^{\prime \prime}(s)\right) d s, \quad 0 \leq t \leq 1 .
\end{aligned}
$$

which starts off with the zero function.

In Figures 1 and 2 below, we will illustrate the first several elements in the schemes. The figures demonstrate the iterative process.

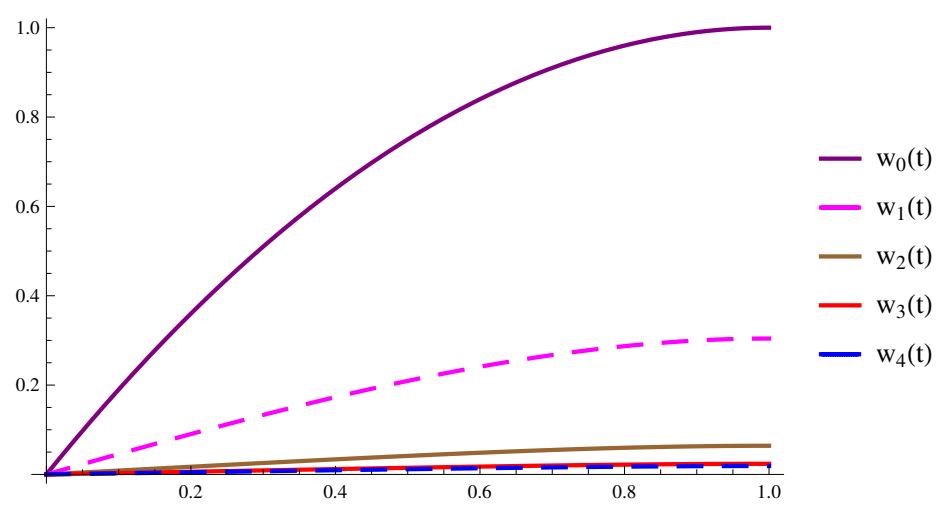

Figure 1. Curves of successive iterations.

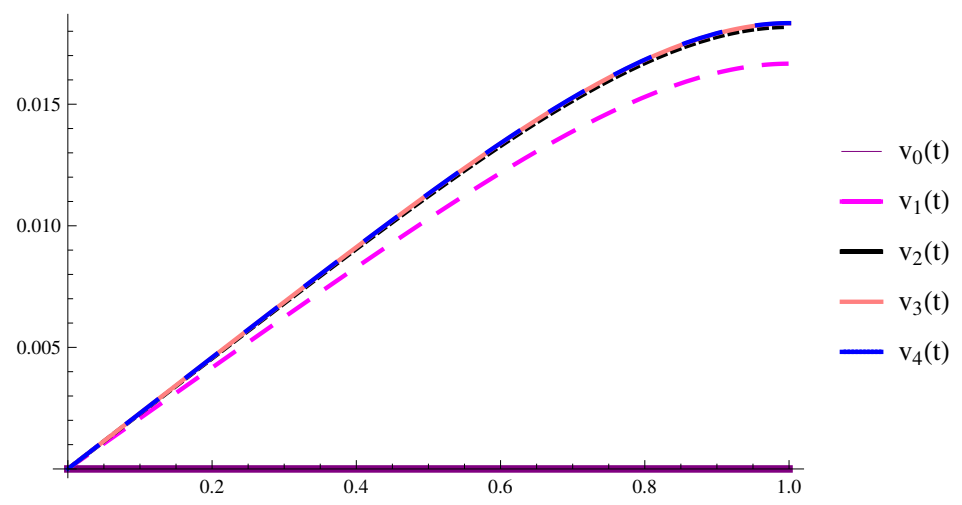

Figure 2. Curves of successive iterations.

and

$$
\begin{aligned}
& \left\|w_{1}-w_{0}\right\|=2.000000000000000000, \quad\left\|v_{1}-v_{0}\right\|=0.0833333333333333333, \\
& \left\|w_{2}-w_{1}\right\|=0.657289255401234568, \quad\left\|v_{2}-v_{1}\right\|=0.0076774691358024691 \text {, } \\
& \left\|w_{3}-w_{2}\right\|=0.144307032230180722, \quad\left\|v_{3}-v_{2}\right\|=0.0007056973781479649 \text {, } \\
& \left\|w_{4}-w_{3}\right\|=0.020648460036251130, \quad\left\|v_{4}-v_{3}\right\|=0.0000666371356413746 \text {. }
\end{aligned}
$$


Funding: This work was supported by the National Natural Science Foundation of China (NO.11126245), China Scholarship Council (CSC-[2014]3072), Emerging Interdisciplinary Project of Central University of Finance and Economics, and Discipline Construction Fund of the Central University of Finance and Economics.

Institutional Review Board Statement: Not applicable.

Informed Consent Statement: Not applicable.

Data Availability Statement: Not applicable.

Conflicts of Interest: 'The author declares no conflict of interest.

\section{References}

1. Padhi, S.; Pati, S. Theory of Third-Order Differential Equations; Springer: Berlin/Heidelberg, Germany, 2013.

2. Yao, Q.; Feng, Y. The Existence of Solution for a Third-Order Two-Point Boundary Value Problem. Appl. Math. Lett. 2002, 15, 227-232. [CrossRef]

3. Feng, Y.; Liu, S. Solvability of a third-order two-point boundary value problem. Appl. Math. Lett. 2005, 18, 1034-1040. [CrossRef]

4. Li, S. Positive solutions of nonlinear singular third-order two-point boundary value problem. J. Math. Anal. Appl. 2006, 323, 413-425. [CrossRef]

5. Hopkins, B.; Kosmatov, N. Third-order boundary value problems with sign-changing solutions. Nonlinear Anal. 2007, 67, 126-137. [CrossRef]

6. Gao, Y.; Wang, F. Existence of Solutions of Nonlinear Mixed Two-Point Boundary Value Problems for Third-Order Nonlinear Differential Equation. J. Appl. Math. 2012, 2012, 262139. [CrossRef]

7. Sun, Y.; Zhao, M.; Li, S. Monotone positive solution of nonlinear third-order two-point boundary value problem. Miskolc Math. Notes 2014, 15, 743-752. [CrossRef]

8. Kelevedjiev, P.; Todorov, T. Existence of solutions of nonlinear third-order two-point boundary value problems. Electron. J. Qual. Theory Differ. Equ. 2019, 23, 1-15. [CrossRef]

9. Ge, Q.; Zhang, X. Numerical Solution for Third-Order Two-Point Boundary Value Problems with the Barycentric Rational Interpolation Collocation Method. J. Math. 2021, 2021, 6698615.

10. Amann, H. Fixed point equations and nonlinear eigenvalue problems in order Banach spaces. SIAM Rev. 1976, 18, 620-709. [CrossRef]

11. Jiang, D.; Agarwal, R.P. A uniqueness and existence theorem for a singular third-order boundary value problem on $[0, \infty)$. Appl. Math. Lett. 2002, 15, 445-451. [CrossRef]

12. Du, Z.; Ge, W.; Lin, X. Existence of solutions for a class of third-order nonlinear boundary value problems. J. Math. Anal. Appl. 2004, 294, 104-112. [CrossRef]

13. Li, H.; Feng, Y.; Bu, C. Non-conjugate boundary value problem of a third order differential equation. Electron. J. Qual. Theory Differ. Equ. 2015, 21, 1-19. [CrossRef]

14. Sun, Y. Positive solutions for one-dimensional third-order $p$-Laplacian boundary value problems. Adv. Differ. Equ. 2017, 95, 1089-1099. [CrossRef]

15. Ugurlu, E. Regular third-order boundary value problems. Appl. Math. Comput. 2019, 343, 247-257.

16. Ramos, H.; Rufai, M.A. A two-step hybrid block method with fourth derivatives for solving third-order boundary value problems. J. Comput. Appl. Math. 2021, in press. [CrossRef]

17. Ma, D.; Du, Z.; Ge, W. Existence and iteration of monotone positive solutions for multipoint boundary value problems with p-Laplacian operator. Comput. Math. Appl. 2005, 50, 729-739. [CrossRef]

18. Sun, B.; Ge, W. Successive iteration and positive pseudo-symmetric solutions for a three-point second-order $p$-Laplacian boundary value problems. Appl. Math. Comput. 2007, 188, 1772-1779. [CrossRef]

19. Sun, B.; Ge, W. Existence and iteration of positive solutions for some $p$-Laplacian boundary value problems. Nonlinear Anal. 2007, 67, 1820-1830. [CrossRef]

20. Ladde, G.; Lakshmikantham, V.; Vatsala, A. Monotone Iterative Techniques for Nonlinear Differential Equations; Pitman: Boston, MA, USA, 1985.

21. Nieto, J.; Jiang, Y.; Jurang, Y. Monotone iterative method for functional differential equations. Nonlinear Anal. $1998,32,741-747$. [CrossRef] 